\title{
A RETROSPECTIVE EVALUATION OF MEAN PLATELET VOLUME AS A DISCRIMINATING FACTOR IN THROMBOCYTOPENIA OF HYPOPRODUCTIVE AND HYPERDESTRUCTIVE AETIOLOGIES
}

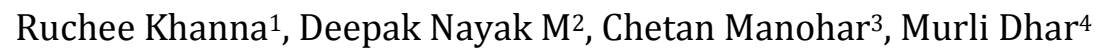

\section{HOW TO CITE THIS ARTICLE:}

Ruchee Khanna, Deepak Nayak M, Chetan Manohar, Murli Dhar. "A retrospective evaluation of mean platelet volume as a discriminating factor in thrombocytopenia of hypoproductive and hyperdestructive aetiologies". Journal of Evolution of Medical and Dental Sciences 2013; Vol. 2, Issue 47, November 25; Page: 9059-9065.

ABSTRACT: BACKGROUND: Bone marrow examination is the gold standard test in determining the cause of thrombocytopenia; whether it is of hypoproductive or hyperdestructive nature. However, this process has distinct disadvantages such as being invasive, not feasible in critical situations and is often not preferred by clinicians and patients alike. The introduction of platelet indices in automated hematology analyzers has provided a small window into the cause of thrombocytopenias in the recent years. Mean platelet volume (MPV) has evoked much interest in this regard. This study aimed at assessing the predictive value of MPV in determining the aetiology of thrombocytopenia. MATERIALS AND METHODS: We analyzed 100 cases of thrombocytopenia (platelet count < $100 \times 10^{3}$ / cu.mm) on a retrospective basis for a period of one month. The cases were divided into 2 groups: with and without bone marrow diseases; based on individual pathophysiology. A statistical analysis was done to assess predictive values, odds ratio and confidence intervals and also to elicit a cut-off value for MPV. RESULTS: The mean MPV in the group with marrow disease was 8.95fl while the group without marrow disease was 9.42fl. The difference in MPV between the 2 groups was not statistically significant ( $P$ value: 0.088). The sensitivity and specificity scores as tabulated by the receptor operating characteristic (ROC) curve at a cut-off of $9.35 \mathrm{fl}$ were $69 \%$ and $55 \%$ respectively. CONCLUSIONS: The study concluded that although MPV may provide a small initial insight into the aetiology of thrombocytopenia, it is limited by insufficient sensitivity and specificity. A bone marrow examination continues to be the gold standard to differentiate the hypoproductive and hyperdestructive thrombocytopenias.

KEY WORDS: Mean platelet volume, thrombocytopenia, bone marrow disease

INTRODUCTION: Many a clinician would agree that thrombocytopenia is one of the most critical and difficult clinical conditions to manage. Hence the need for a platelet count has increased over the years in most clinical laboratories. However, the information available on these small particles is not restricted to the counts alone. The data on the size of the platelets have interested physicians since the last three decades. ${ }^{1-3}$ The recent advancements in automated hematology analyzers have added new dimensions to the platelet indices (mean platelet volume, plateletcrit, platelet distribution width and platelet -large cell ratio). While the data on red blood size (mean corpuscular volume) is universally acknowledged and even applied, the diagnostic and prognostic utility of platelet size (mean platelet volume) in thrombocytopenia is still in its nascent stage of exploration. The recent years has seen a number of studies on mean platelet volume (MPV) as a factor to discriminate the cause of thrombocytopenia (Bowles et $\mathrm{al}^{4}$ and Numbenjapon et $\mathrm{al}^{5}$ ). Levine, et $\mathrm{al}^{6}$ had proposed that the mechanism of thrombocytopenia could be simplified into two groups after excluding a splenic sequestration: hyperdestructive and hypoproductive.For a long time the bone marrow examination 


\section{ORIGINAL ARTICLE}

has remained the gold standard test in diagnosing the cause for thrombocytopenia. However, this procedure is invasive, time consuming and has to be diligently examined by a hematopathologist. Moreover, it also may not be feasible in critical thrombocytopenias where the risk of a bleeding diathesis may be overt; meriting a lack of consent from both clinicians and patients alike.

The MPV is a simple, non-invasive and reproducible method available in most healthcare centers, courtesy; the modern hematology cell analyzers.These machines measure the platelet count by impedance method. The data also computes the MPV. This study was conducted to assess and statistically analyze the role of MPV as discriminating factor in thrombocytopenias which involves the bone marrow (decreased platelet production/ hypoproductive) and in cases where the marrow is normal (peripheral destruction of platelets/ hyperdestructive).

MATERIALS AND METHODS: A retrospective study of 100 cases was undertaken in the Clinical Laboratory and Hematology division of Kasturba Hospital, Manipal. The study was conducted after obtaining the approval from the Ethics Committee, Kasturba Hospital, Manipal. The cases included were thrombocytopenias (defined by platelet count $<100 \times 10^{3} /$ cu.mm.) selected from the software data on the LH 750 cell analyzer (Beckman-Fullerton) for the month of April 2013. The cases where the bone marrow was performed were also included. The blood samples were collected in ethylene diamine tetra-acetic acid (EDTA) anticoagulant vacutainers. The blood samples were subjected to a complete blood count analysis using LH 750 cell counter (Beckman-Fullerton). This is a five-part differential cell analyzer which gives a platelet count by impedance method. As platelets pass via an aperture by a streamlined flow, an electronic impulse is generated due to the alteration in the current flow between the electrodes. The amplitude of the pulse generated is proportional to the volume (MPV) and the number of pulses gives the platelet count. The reference range for MPV in normal subjects in this analyzer is 7-11fl. All the samples were analyzed within 3 hours of venipuncture to eliminate the possibility of platelet swelling in EDTA. The platelet count and the corresponding MPV of the 100 cases were recorded. The platelet count was confirmed under oil immersion field. Two hematopathologists who were blinded to the clinical diagnosis and MPV reported on the platelet counts. All bone marrow aspirates and biopsies were reported independently.

The thrombocytopenia was categorized into 2 main groups: Group A where thrombocytopenia showed a bone marrow disease and Group B where the thrombocytopenia was due to peripheral destruction. The group A yielded 26 cases while the group B had 76 cases.

Statistical analysis: The statistics were carried out using the SPSS software version 16.0 for windows (Chicago, IL, USA). Results were expressed as mean +/- 2 SD along with the range. Student's t test was applied to compare the parameters between groups A and B. P value $<0.05$ was considered artistically significant. The positive and negative predictive values, odds ratio and confidence intervals were tabulated. The sensitivity and specificity of MPV as predictors of marrow disease were assessed by performing receptor operating characteristic (ROC) curve analysis.

RESULTS: Of the 100 cases studied, bone marrow disease was diagnosed in 26 cases (group A) while 74 cases did not have a marrow disease (group B). Table 1 shows different aetiologies for thrombocytopenia for all the 100 cases. 


\section{ORIGINAL ARTICLE}

Table 2 shows the number of cases in group A (with bone marrow disease) along with their mean MPV. Mean MPV suggests the mean of all the individual MPV in the 26 cases in group A. Acute lymphoblastic leukemia constituted the most number of cases, followed by megaloblastic anemia. All the six disease categories had a variable mean MPV with megaloblastic anemia having the highest mean MPV (9.68fl).

Table 3 shows the number of cases in group B (without bone marrow disease) along with their respective mean MPV. It was seen that all categories except malaria had a mean MPV more than $9 \mathrm{fl}$. The mean MPV in cases of bone marrow disease (26 cases) was 8.95fl (SD: 1.07; range: 7.88-10.02 fl). The mean MPV in cases without bone marrow disease (74 cases) was 9.42fl (SD: 1.22; range: $8.22-10.64 \mathrm{fl}$ ). The student's t test showed a $\mathrm{P}$ value of 0.088 ; which was not statistically significant.

Table 4 demonstrates the comparison of negative predictive value for bone marrow disease at different MPV threshold levels. The higher the MPV, greater is the discriminant function for excluding bone marrow disease in patients with thrombocytopenia. A MPV of more than $12 \mathrm{fl}$ has a negative predictive value of $100 \%$. It also gradually declined as the MPV threshold decreased. Table 6 shows the comparison of positive predictive value of the MPV for marrow disease at different threshold levels. It was observed that the positive predictive value was variable when compared with different MPV thresholds and showed no particular pattern.

The analysis of ROC curve showed that a cut-off of $9.35 \mathrm{fl}$ yielded a sensitivity of $69 \%$ and a specificity of $55 \%$ (figure 1 )

DISCUSSION: Today's clinical medicine has taken many important strides and physicians are increasingly dependent on rapid laboratory tests to assess the risk of a specific disease entity. These diagnostic algorithms have partially or completely eclipsed conventional methods of diagnosis. Specific examples include Troponin levels in ischemic heart disease ${ }^{7}$ and D-dimer assay in hemolytic anemia and venous thromboembolism ${ }^{8}$. With reference to cell counters, the modern automated analyzers have aided in providing new parameters which are useful in rapid testing of diseases. MPV has been put to test by researchers in the recent years; yielding consistent results in distinguishing the two types of thrombocytopenia. ${ }^{4,10,11}$ However, the wider utility of this factor has yielded variable results. ${ }^{4,5,12}$ In the present study, the cases with thrombocytopenia due to a bone marrow disease had a lower mean MPV while the other group without marrow involvement had a higher mean MPV. However, the difference between the groups was statistically not significant.

It was noted in the present study that the cases with leukemias which are associated with marrow suppression had variable mean MPVs. The acute lymphoblastic leukemias and chronic leukemias had a mean MPV less than 9fl. In contrast, the acute myeloid leukemias had a higher mean MPV. The MPV in these cases were noted at the time of marrow diagnosis so that chemotherapy drugsdid not affect the test results. When the negative predictive value for bone marrow disease was analyzed at different MPV threshold levels, it was noted that an increase was in parallel with increasing MPV threshold. But a similar correlation did not exist with positive predictive value. This data hints that the positive predictivity for bone marrow disease by MPV was not reliable. The analysis of sensitivity and specificity of MPV was also not diagnostic as an indicator of marrow disease at a cut-off of $9.35 \mathrm{fl}$ (sensitivity- 69\% and specificity- 55\%). Similar results were acknowledged by the studies conducted by Chandra et $\mathrm{al}^{12}$. 


\section{ORIGINAL ARTICLE}

The direction of research to determine the hypoproductive and hyperdestructive thrombocytopenia has veered towards other platelet indices Borkataky et alhave demonstrated that platelet distribution width (PDW) has discriminating property to determine the aetiology of thrombocytopenia. ${ }^{13}$ Other researchers such as Niethammer et al have upheld the diagnostic value of platelet histogram maximum rather than MPV to differentiate between idiopathic thrombocytopenic purpura and hypoproductive thrombocytopenia. ${ }^{14} \mathrm{Ntaios}$ et al have found a combination of MPV and PDW superior to platelet-large cell ratio (P-LCR). ${ }^{15}$ The present study is not without its limitations. The sample size was a small one and secondly, we did not analyze the other platelet indices apart from MPV, such as PDW. This was done intently as we wanted to test only a single parameter. Also, P-LCR was not provided by the autoanalyzer. Future research in this regard by binding these indices may lead to more definitive answers. A different approach was adopted by Kurata et al, who assessed reticulated platelets which are newly released from the marrow. ${ }^{16}$ By using RNA-binding dyes and flow cytometric analysis, these immature platelets were successful as a discriminating guide to delineate the aetiology of thrombocytopenia. However, this test is expensive, requires extensive quality control measures and is relatively time consuming.

Few disclaimers have to be considered while one interprets MPV in isolation: a spurious increase in MPV contributed by red blood cell fragmentation which can be counted by the analyzer as a giant platelet, a combination of hypoproductive and hyperdestructive thrombocytopenia and splenic sequestration; where large platelets are entrapped in spleen but not released into the circulation. ${ }^{10,17.18}$

CONCLUSION: MPV is easily available in most five part differential counters and may provide a small initial window into the aetiology of thrombocytopenia. But it is limited by insufficient sensitivity and specificity. A bone marrow examination continues to be the gold standard to differentiate the hypoproductive and hyperdestructive thrombocytopenias. A combination of all the platelet indices and new parameters such as reticulated platelets and immature platelet fraction may be the way ahead to predict the cause of thrombocytopenia.

\section{REFERENCES:}

1. Giles C. The platelet count and mean platelet volume. Br. J. Hematol 1981; 48:31-37.

2. Reardon D.M., Hutchinson D, Preston F.E., Trowbridge E.A. The routine measurement of platelet volume: a comparison of aperture -impedance and flow cytometric systems. Clin. Lab Haem. 1985; 7: 251-257.

3. Trowbridge E.A., Reardon D.M., Hutchinson D, Pickering C. The routine measurement of platelet volume: a comparison of light-scattering and aperture -impedance technologies. Clinical Physics and Physiological Measurements 1985;6:221-238.

4. Bowles K.M., Cooke L.J., Richards E.M. Baglin T.P. Platelet size has diagnostic value in patients with thrombocytopenia. Clin. Lab Haem.2005;27:370-373.

5. NumbenjaponT.et al. A prospective evaluation of normal mean platelet volume in discriminating hyperdestructive thrombocytopenia from hypoproductive thrombocytopenia. Intl. Jnl. Lab. Hem.2008:30:408-414.

6. Levine S.P. Thrombocytopenia: pathophysiology and classification. In: Wintrobe's Clinical Hematology. Lee G.R,et al (eds),9th edn..William \& Wilkins, Baltimore, USA.1999: pp.15791582. 
7. George M.R. Thrombocytopenia: pathophysiology and classification. In: Wintrobe's Clinical Hematology.John P.G.,et al(eds), 12th edn. Lippincott William \& Wilkins, Philadelphia, USA.2009: pp.1289-1291.

8. Hamm C.W., et al.emergency room triage of patients with acute chest pain by means of rapid testing for cardiac troponin T or troponin I. N Eng. Jour. Med.1997;337:1648-1653.

9. Wells P.S.,et al. Evaluation of D-dimer in the diagnosis of suspected deep -vein thrombosis. N Eng. Jour. Med.2003;349:1227-1235.

10. Bessman J.D., Gilmer P.R., Gardner F.H. Use of mean platelet volume improves the detection of platelet disorders. Blood Cells 1985;127-135.

11. Kaito K.,et al.platelet size deviation width, platelet large cell ratio and mean platelet volume have sufficient sensitivity and specificity in the diagnosis of immune thrombocytopenia. Br. J. Hematol. 2005;128:698-702.

12. H.Chanrdra ,et al.Role of mean platelet volume as discriminating guide for bone marrow disease in patients with thrombocytopenia.Intl. Jnl. Lab. Hem.2010; 32:498-505.

13. Borkataky S. et al. Role of platelet volume indices in the differential diagnosis of thrombocytopenia. A simple and inexpensive method. Hematology 2009; 14: 182-186.

14. Niethammer A.G., Forman E.N. use of platelet histogram maximum in evaluating thrombocytopenia. Am J Hematol 1999;60: 19-23.

15. Ntaios G. et al. Increased values of mean platelet volume and platelet size deviation width may provide a safe positive diagnosis of idiopathic thrombocytopenic purpura. Acta Hematologica 2008; 119:173-177.

16. Kurata Y. et al. Diagnostic value of tests for reticulated platelets, plasma glycocalcin and thrombopoietin levels for discriminating between hyperdestructive and hypoplastic thrombocytopenia. Am J Clin Pathol 2001;115:656-664.

17. Garg S.K., Lackner H., Karpatkin S. The increased percentage of megathrombocytes in vaious clinical disorders. Ann. Int. Med.1972;77:361-369.

18. Karpatkin S., Freedman M.L. Hypersplenic thrombocytopenia differentiated from increased peripheral destruction by platelet volume.Ann. Int. Med.1978;89:200-203.

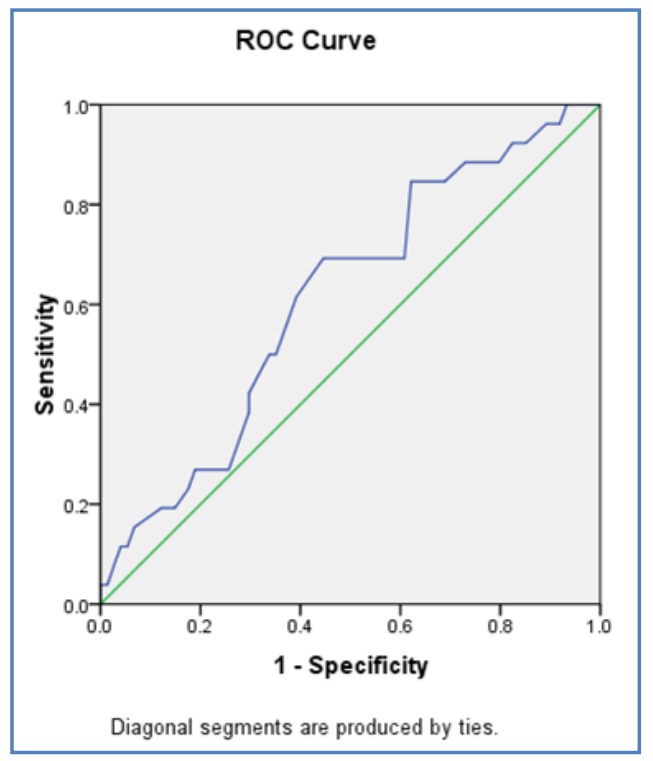

Fig. 1: ROC curve analysis showing a cut-off of $9.35 \mathrm{fl}$ 


\begin{tabular}{|l|c|}
\hline Cause for thrombocytopenia & No. of cases \\
\hline Acute Lymphoblastic Leukemia & 13 \\
Megaloblastic Anemia & 8 \\
Acute Myeloid Leukemia & 2 \\
Aplastic Anemia & 1 \\
Chronic Lymphoproliferative Disease & 1 \\
Chronic Myeloid Leukemia & 1 \\
Sepsis & 40 \\
Liver disease* & 6 \\
Malaria & 6 \\
Dengue & 14 \\
Idiopathic Thrombocytopenic Purpura & 5 \\
Others & 3 \\
\hline *without splenomegaly & \\
\hline Table 1: Causes of thrombocytopenia in all 100 cases \\
\hline
\end{tabular}

\begin{tabular}{|l|c|c|}
\hline \multicolumn{1}{|c|}{ Disease category } & No.of cases & MPV (fl, mean +/- 2sd) \\
\hline Acute Lymphoblastic Leukemia & 13 & $8.44+/-0.92$ \\
Megaloblastic Anemia & 8 & $9.68+/-0.98$ \\
Acute Myeloid Leukemia & 2 & $9.50+/-1.83$ \\
Aplastic Anemia & 1 & - \\
Chronic Lymphoproliferative Disease & 1 & - \\
Chronic Myeloid Leukemia & 1 & - \\
\hline \multicolumn{2}{|r|}{ Table 2: Mean MPV in group A (with bone marrow disease) }
\end{tabular}

\begin{tabular}{|l|c|c|}
\hline \multicolumn{1}{|c|}{ Disease category } & No.of cases & MPV (fl, mean +/- 2sd) \\
\hline Sepsis & 40 & $9.28+/-1.17$ \\
Liver disease & 6 & $9.98+/-2.18$ \\
Malaria & 6 & $8.81+/-1.04$ \\
Dengue & 14 & $9.55+/-1.01$ \\
Idiopathic Thrombocytopenic Purpura & 5 & $10.12+/-1.13$ \\
Others & 3 & $9.60+/-0.42$ \\
\hline \multicolumn{2}{|c|}{ Table 3: Mean MPV in group B (without bone marrow disease) } \\
\hline
\end{tabular}

\begin{tabular}{|c|c|c|c|c|c|c|c|}
\hline \multirow{2}{*}{$\begin{array}{c}\text { MPV } \\
\text { threshold } \\
\text { (fl) }\end{array}$} & \multirow{2}{*}{$\begin{array}{l}\text { No. of } \\
\text { cases }\end{array}$} & \multirow{2}{*}{$\begin{array}{l}\text { No. of pts. without } \\
\text { marrow disease }\end{array}$} & \multirow{2}{*}{$\begin{array}{l}\% \text { of pts in } \\
\text { this group }\end{array}$} & \multirow{2}{*}{$\begin{array}{l}\text { NPV } \\
(\%)\end{array}$} & \multirow{2}{*}{$\begin{array}{l}\text { Odd's } \\
\text { Ratio }\end{array}$} & \multicolumn{2}{|c|}{$95 \%$ CI } \\
\hline & & & & & & Lower & Upper \\
\hline$>12$ & 2 & 2 & 2 & 100 & - & - & - \\
\hline$>11.5$ & 6 & 5 & 6 & 83.3 & 1.811 & 0.201 & 16.272 \\
\hline$>11$ & 8 & 8 & 8 & 100 & - & - & - \\
\hline$>10.5$ & 15 & 13 & 15 & 86.67 & 2.557 & 0.536 & 12.192 \\
\hline
\end{tabular}


ORIGINAL ARTICLE

\begin{tabular}{|c|c|c|c|c|c|c|c|}
\hline$>10$ & 22 & 19 & 22 & 86.36 & 2.648 & 0.713 & 9.828 \\
\hline$>9.5$ & 37 & 29 & 37 & 78.37 & 1.450 & 0.558 & 3.766 \\
\hline$>9$ & 61 & 49 & 61 & 80.32 & 2.286 & 0.921 & 5.676 \\
\hline$>8.5$ & 74 & 53 & 74 & 71.62 & 0.600 & 0.200 & 1.802 \\
\hline$>8$ & 84 & 63 & 84 & 75 & 1.363 & 0.424 & 4.379 \\
\hline$>7.5$ & 93 & 70 & 93 & 75.26 & 2.282 & 0.475 & 10.964 \\
\hline$>7$ & 100 & 74 & 100 & 74 & - & - & - \\
\hline
\end{tabular}

\begin{tabular}{|c|c|c|c|c|c|c|c|}
\hline $\begin{array}{c}\text { MPV } \\
\text { threshold } \\
(\mathrm{fl})\end{array}$ & $\begin{array}{c}\text { No. of } \\
\text { cases }\end{array}$ & $\begin{array}{c}\text { No. of pts. with } \\
\text { marrow disease }\end{array}$ & $\begin{array}{c}\text { \% of pts in } \\
\text { this group }\end{array}$ & $\begin{array}{c}\text { PPV } \\
(\%)\end{array}$ & $\begin{array}{c}\text { Odd's } \\
\text { Ratio }\end{array}$ & \multicolumn{2}{|c|}{ L5\% CI } \\
\hline$<12$ & 98 & 26 & 26 & 26.53 & - & - & - \\
\hline$<11.5$ & 94 & 25 & 25 & 26.59 & 2.61 & 0.305 & 22.313 \\
\hline$<11$ & 92 & 25 & 25 & 27.17 & 1.48 & 0.383 & 5.739 \\
\hline$<10.5$ & 85 & 23 & 23 & 27.05 & 2.64 & 0.713 & 9.828 \\
\hline$<10$ & 78 & 23 & 23 & 29.48 & 1.45 & 0.558 & 3.766 \\
\hline$<9.5$ & 63 & 18 & 18 & 28.57 & 1.84 & 0.746 & 4.563 \\
\hline$<9$ & 39 & 13 & 13 & 33.33 & 1.06 & 0.387 & 2.932 \\
\hline$<8.5$ & 26 & 7 & 7 & 26.92 & 1.36 & 0.424 & 4.379 \\
\hline$<8$ & 16 & 5 & 5 & 31.25 & 2.28 & 0.475 & 22.313 \\
\hline$<7.5$ & 7 & 3 & 3 & 42.85 & - & - & - \\
\hline$<7$ & 0 & 0 & 0 & - & - & - & - \\
\hline
\end{tabular}

\section{AUTHORS:}

1. Ruchee Khanna

2. Deepak Nayak M.

3. Chetan Manohar

4. Murli Dhar

\section{PARTICULARS OF CONTRIBUTORS:}

1. Associate Professor, Department of Pathology, Kasturba Medical College, Manipal, Manipal University.

2. Assistant Professor, Department of Pathology, Kasturba Medical College, Manipal, Manipal University.

3. Professor, Department of Pathology, Kasturba Medical College, Manipal, Manipal University.
4. Associate Professor, Department of Statistics, Manipal University.

\section{NAME ADDRESS EMAIL ID OF THE CORRESPONDING AUTHOR:}

Dr. Ruchee Khanna,

Associate Professor,

Department of Pathology,

Kasturba Medical College, Manipal,

Manipal University.

Email - drruchi2003@yahoo.com

Date of Submission: 04/11/2013.

Date of Peer Review: 06/11/2013.

Date of Acceptance: 12/11/2013.

Date of Publishing: 19/11/2013 